\title{
On a Robin problem with $p$-Laplacian and reaction bounded only from above
}

\author{
Salvatore A. Marano* \\ Dipartimento di Matematica e Informatica, Università degli Studi di Catania, \\ Viale A. Doria 6, 95125 Catania, Italy \\ E-mail:marano@dmi.unict.it \\ Nikolaos S. Papageorgiou \\ Department of Mathematics, National Technical University of Athens, \\ Zografou Campus, Athens 15780, Greece \\ E-mail:npapg@math.ntua.gr
}

\begin{abstract}
The existence of three smooth solutions, one negative, one positive, and one nodal, to a homogeneous Robin problem with $p$-Laplacian and Carathéodory reaction is established. No sub-critical growth condition is taken on. Proofs exploit variational as well as truncation techniques. The case $p=2$ is separately examined, obtaining a further nodal solution via Morse's theory.
\end{abstract}

Keywords: p-Laplacian, Robin problem, constant-sign solution, nodal solution

AMS Subject Classification: 35J20, 35J60, 35J92

\section{Introduction}

Let $\Omega$ be a bounded domain in $\mathbb{R}^{N}$ with a smooth boundary $\partial \Omega$, let $1<p<\infty$, let $f: \Omega \times \mathbb{R} \rightarrow \mathbb{R}$ be a Carathéodory function, and let $\beta \in C^{0, \alpha}\left(\partial \Omega, \mathbb{R}_{0}^{+}\right)$for some $\alpha \in(0,1)$.

\footnotetext{
${ }^{*}$ Corresponding author
} 
Consider the homogeneous Robin problem

$$
\begin{cases}-\Delta_{p} u=f(x, u) & \text { in } \Omega \\ \frac{\partial u}{\partial n_{p}}+\beta(x)|u|^{p-2} u=0 & \text { on } \partial \Omega\end{cases}
$$

where $\Delta_{p}$ denotes the $p$-Laplace differential operator, namely $\Delta_{p} u:=\operatorname{div}\left(|\nabla u|^{p-2} \nabla u\right)$ for all $u \in W^{1, p}(\Omega)$, while $\frac{\partial u}{\partial n_{p}}:=|\nabla u|^{p-2} \nabla u \cdot n$, with $n(x)$ being the outward unit normal vector to $\partial \Omega$ at its point $x$. As in [12, p. 1066], $u \in W^{1, p}(\Omega)$ is called a (weak) solution of (1.1) provided

$$
\int_{\Omega}|\nabla u|^{p-2} \nabla u \cdot \nabla v d x+\int_{\partial \Omega} \beta|u|^{p-2} u v d \sigma=\int_{\Omega} f(x, u) v d x \quad \forall v \in W^{1, p}(\Omega) .
$$

Equations driven by $p$-Laplacian type operators have been widely investigated under Dirichlet boundary conditions, mainly through variational, sub-super-solutions, and truncation techniques [18, 6, 7], besides Morse's theory [23]. There is a wealth of good results and the relevant literature looks daily increasing. On the other hand, these methods cannot always be adapted in a simple way to treat Neumann (i.e., $\beta \equiv 0$ ), or more generally Robin $(\beta \not \equiv 0)$, problems. That's why over the last few years the study of (1.1) has been receiving attention and very nice papers are already available. The more close to our work are [5, 11, 19] and, above all, [22]. Indeed, here, we prove the existence of three $C^{1}$ solutions to Problem (1.1), one positive, one negative, and one nodal, without assuming that $t \mapsto f(x, t)$ exhibits a sub-critical behavior but is merely bounded on bounded sets. Moreover, roughly speaking, we suppose that

$$
\limsup _{t \rightarrow \pm \infty} \frac{f(x, t)}{|t|^{p-2} t} \leq a_{0}<\lambda_{1} \text { and } \lambda_{2}<a_{1} \leq \liminf _{t \rightarrow 0} \frac{f(x, t)}{|t|^{p-2} t} \leq \limsup _{t \rightarrow 0} \frac{f(x, t)}{|t|^{p-2} t} \leq a_{2}<+\infty
$$

uniformly in $x \in \Omega$, with $\lambda_{1}$ (respectively, $\lambda_{2}$ ) being the first (respectively, second) eigenvalue of $\left(-\Delta_{p}, W^{1, p}(\Omega)\right)$ under Robin's boundary condition; see Section 3 for precise formulations. So, no global growth from below is imposed on $t \mapsto f(x, t)$. The meaningful special case

$$
f(x, t):=\lambda|t|^{p-2} t-g(x, t) . \quad(x, t) \in \Omega \times \mathbb{R},
$$

where $\lambda>\lambda_{2}$, is also examined and some results of [22] extended; cf. also [5, 11, 19], which however require $\beta \equiv 0$. When $p=2$ we obtain a second nodal solution by assuming, among other things, $f(x, \cdot) \in C^{1}(\mathbb{R})$ and

$$
\left|f_{t}^{\prime}(x, t)\right| \leq a_{3}\left(1+|t|^{r-2}\right) \quad \forall(x, t) \in \Omega \times \mathbb{R},
$$


with $2 \leq r<2^{*}$. Let us finally point out that an analogous investigation might be performed for the problem

$$
-\Delta_{p} u+a(x)|u|^{p-2} u=f(x, u) \quad \text { in } \quad \Omega, \quad \frac{\partial u}{\partial n_{p}}=0 \quad \text { on } \quad \partial \Omega,
$$

where $a \in L^{\infty}(\Omega)$ may change sign, exploiting the results of [20].

\section{Preliminaries}

Let $(X,\|\cdot\|)$ be a real Banach space. Given a set $V \subseteq X$, write $\bar{V}$ for the closure of $V$, $\partial V$ for the boundary of $V$, and $\operatorname{int}_{X}(V)$ or simply $\operatorname{int}(V)$, when no confusion can arise, for the interior of $V$. If $x \in X$ and $\delta>0$ then

$$
B_{\delta}(x):=\{z \in X:\|z-x\|<\delta\} .
$$

The symbol $\left(X^{*},\|\cdot\|_{X^{*}}\right)$ denotes the dual space of $X,\langle\cdot, \cdot\rangle$ indicates the duality pairing between $X$ and $X^{*}$, while $x_{n} \rightarrow x$ (respectively, $x_{n} \rightarrow x$ ) in $X$ means 'the sequence $\left\{x_{n}\right\}$ converges strongly (respectively, weakly) in $X^{\prime}$.

Let $T$ be a topological space and let $L$ be a multifunction from $T$ into $X$ (briefly, $\left.L: T \rightarrow 2^{X}\right)$, namely a function which assigns to each $t \in T$ a nonempty subset $L(t)$ of $X$. We say that $L$ is lower semi-continuous when $\{t \in T: L(t) \cap V \neq \emptyset\}$ turns out to be open in $T$ for every open set $V \subseteq X$. A function $l: T \rightarrow X$ is called a selection of $L$ provided $l(t) \in L(t)$ for all $t \in T$.

We say that $\Phi: X \rightarrow \mathbb{R}$ is coercive iff

$$
\lim _{\|x\| \rightarrow+\infty} \Phi(x)=+\infty
$$

while $\Phi$ is called weakly sequentially lower semi-continuous when $x_{n} \rightarrow x$ in $X$ implies $\Phi(x) \leq \liminf _{n \rightarrow \infty} \Phi\left(x_{n}\right)$. Let $\Phi \in C^{1}(X)$. The classical Palais-Smale compactness condition for $\Phi$ reads as follows.

(PS) Every sequence $\left\{x_{n}\right\} \subseteq X$ such that $\left\{\Phi\left(x_{n}\right)\right\}$ is bounded and $\left\|\Phi^{\prime}\left(x_{n}\right)\right\|_{X^{*}} \rightarrow 0$ has a convergent subsequence.

Define, provided $c \in \mathbb{R}$,

$$
\Phi^{c}:=\{x \in X: \Phi(x) \leq c\}, \quad K_{c}(\Phi):=K(\Phi) \cap \Phi^{-1}(c)
$$

where, as usual, $K(\Phi)$ denotes the critical set of $\Phi$, i.e., $K(\Phi):=\left\{x \in X: \Phi^{\prime}(x)=0\right\}$. 
We say that $A: X \rightarrow X^{*}$ is of type $(\mathrm{S})_{+}$iff

$$
x_{n} \rightarrow x \quad \text { in } \mathrm{X}, \quad \limsup _{n \rightarrow+\infty}\left\langle A\left(x_{n}\right), x_{n}-x\right\rangle \leq 0
$$

imply $x_{n} \rightarrow x$. The next elementary result [15, Proposition 2.2] will be employed later.

Proposition 2.1. Let $X$ be reflexive and let $\Phi \in C^{1}(X)$ be coercive. Assume $\Phi^{\prime}=A+B$, with $A: X \rightarrow X^{*}$ of type $(\mathrm{S})_{+}$and $B: X \rightarrow X^{*}$ compact. Then $\Phi$ satisfies (PS).

Given a topological pair $(A, B)$ fulfilling $B \subset A \subseteq X$, the symbol $H_{q}(A, B), q \in \mathbb{N}_{0}$, indicates the $\mathrm{q}^{\text {th }}$-relative singular homology group of $(A, B)$ with integer coefficients. If $x_{0} \in K_{c}(\Phi)$ is an isolated point of $K(\Phi)$ then

$$
C_{q}\left(\Phi, x_{0}\right):=H_{q}\left(\Phi^{c} \cap V, \Phi^{c} \cap V \backslash\left\{x_{0}\right\}\right), \quad q \in \mathbb{N}_{0},
$$

are the critical groups of $\Phi$ at $x_{0}$. Here, $V$ stands for any neighborhood of $x_{0}$ such that $K(\Phi) \cap \Phi^{c} \cap V=\left\{x_{0}\right\}$. By excision, this definition does not depend on the choice of $V$. Suppose $\Phi$ satisfies Condition (PS), $\left.\Phi\right|_{K(\Phi)}$ is bounded below, and $c<\inf _{x \in K(\Phi)} \Phi(x)$. Put

$$
C_{q}(\Phi, \infty):=H_{q}\left(X, \Phi^{c}\right), \quad q \in \mathbb{N}_{0}
$$

The second deformation lemma [10, Theorem 5.1.33] implies that this definition does not depend on the choice of $c$. If $K(\Phi)$ is finite, then setting

$$
M(t, x):=\sum_{q=0}^{+\infty} \operatorname{rank} C_{q}(\Phi, x) t^{q}, \quad P(t, \infty):=\sum_{q=0}^{+\infty} \operatorname{rank} C_{q}(\Phi, \infty) t^{q} \quad \forall(t, x) \in \mathbb{R} \times K(\Phi),
$$

the following Morse relation holds:

$$
\sum_{x \in K(\Phi)} M(t, x)=P(t, \infty)+(1+t) Q(t)
$$

where $Q(t)$ denotes a formal series with nonnegative integer coefficients; see for instance [18, Theorem 6.62].

Now, let $X$ be a Hilbert space, let $x \in K(\Phi)$, and let $\Phi$ be $C^{2}$ in a neighborhood of $x$. If $\Phi^{\prime \prime}(x)$ turns out to be invertible, then $x$ is called non-degenerate. The Morse index $d$ of $x$ is the supremum of the dimensions of the vector subspaces of $X$ on which $\Phi^{\prime \prime}(x)$ turns out to be negative definite. When $x$ is non-degenerate and with Morse index $d$ one has

$$
C_{q}(\Phi, x)=\delta_{q, d} \mathbb{Z}, \quad q \in \mathbb{N}_{0}
$$

The monographs [16, 18] represent general references on the subject. 
Throughout the paper, $\Omega$ denotes a bounded domain of the real euclidean $N$-space $\left(\mathbb{R}^{N},|\cdot|\right)$ whose boundary is $C^{2}$ while $\beta \in C^{0, \alpha}\left(\partial \Omega, \mathbb{R}_{0}^{+}\right)$for some $\alpha \in(0,1)$ and $\beta \not \equiv 0$. On $\partial \Omega$ we will employ the $(N-1)$-dimensional Hausdorff measure $\sigma$. The symbol $m$ stands for the Lebesgue measure, $p \in(1,+\infty), p^{\prime}:=p /(p-1),\|\cdot\|_{q}$ with $q \geq 1$ indicates the usual norm of $L^{q}(\Omega), X:=W^{1, p}(\Omega)$, and

$$
\begin{gathered}
\|u\|:=\left(\|\nabla u\|_{p}^{p}+\|u\|_{p}^{p}\right)^{1 / p}, \quad u \in X, \\
C_{+}:=\left\{u \in C^{0}(\bar{\Omega}): u(x) \geq 0 \quad \forall x \in \bar{\Omega}\right\} .
\end{gathered}
$$

Write $p^{*}$ for the critical exponent of the Sobolev embedding $W^{1, p}(\Omega) \subseteq L^{q}(\Omega)$. Recall that $p^{*}=N p /(N-p)$ if $p<N, p^{*}=+\infty$ otherwise, and the embedding is compact whenever $1 \leq q<p^{*}$. Moreover,

$$
\operatorname{int}\left(C_{+}\right)=\left\{u \in C_{+}: u(x)>0 \quad \forall x \in \bar{\Omega}\right\} .
$$

Given $t \in \mathbb{R}, u, v: \Omega \rightarrow \mathbb{R}$, and $f: \Omega \times \mathbb{R} \rightarrow \mathbb{R}$, define

$$
t^{ \pm}:=\max \{ \pm t, 0\}, \quad u^{ \pm}(x):=u(x)^{ \pm}, \quad N_{f}(u)(x):=f(x, u(x)) .
$$

The symbol $u \leq v$ means $u(x) \leq v(x)$ for almost every $x \in \Omega$. If $u, v$ belong to a function space $Y$ and $u \leq v$ then we set

$$
[u, v]:=\{w \in Y: u \leq w \leq v\}
$$

Let $A_{p}: X \rightarrow X^{*}$ be the nonlinear operator stemming from the negative $p$-Laplacian $\Delta_{p}$, i.e.,

$$
\left\langle A_{p}(u), v\right\rangle:=\int_{\Omega}|\nabla u(x)|^{p-2} \nabla u(x) \cdot \nabla v(x) d x \quad \forall u, v \in X .
$$

A standard argument [18, Proposition 2.71] ensures that $A_{p}$ is of type $(\mathrm{S})_{+}$.

Remark 2.1. Given $u \in X$ and $w \in L^{p^{\prime}}(\Omega)$, the condition

$$
\left\langle A_{p}(u), v\right\rangle+\int_{\partial \Omega} \beta(x)|u(x)|^{p-2} u(x) v(x) d \sigma=\int_{\Omega} w(x) v(x) d x, \quad v \in X,
$$

is equivalent to

$$
-\Delta_{p} u=w \quad \text { in } \quad \Omega, \quad \frac{\partial u}{\partial n_{p}}+\beta(x)|u|^{p-2} u=0 \quad \text { on } \quad \partial \Omega .
$$

This easily comes out from the nonlinear Green's identity [10, Theorem 2.4.54]; see for instance the proof of [22, Proposition 3]. 
We shall employ some facts on the spectrum $\sigma\left(-\Delta_{p}\right)$ of the operator $-\Delta_{p}$ with homogeneous Robin boundary conditions. So, consider the nonlinear eigenvalue problem

$$
\begin{cases}-\Delta_{p} u=\lambda|u|^{p-2} u & \text { in } \Omega, \\ \frac{\partial u}{\partial n_{p}}+\beta(x)|u|^{p-2} u=0 & \text { on } \partial \Omega .\end{cases}
$$

The Liusternik-Schnirelman theory provides a strictly increasing sequence $\left\{\lambda_{n}\right\} \subseteq \mathbb{R}_{0}^{+}$of eigenvalues for (2.3). Denote by $E\left(\lambda_{n}\right)$ the eigenspace corresponding to $\lambda_{n}, n \in \mathbb{N}$. From [12, 22] we know that:

$\left(\mathrm{p}_{1}\right) \lambda_{1}$ is positive, isolated, and simple. Further,

$$
\lambda_{1}=\inf \left\{\frac{\|\nabla u\|_{p}^{p}+\int_{\partial \Omega} \beta|u|^{p} d \sigma}{\|u\|_{p}^{p}}: u \in X, u \neq 0\right\} .
$$

$\left(\mathrm{p}_{2}\right)$ There exists an $L^{p}$-normalized eigenfunction $\hat{u}_{1} \in \operatorname{int}\left(C_{+}\right)$associated with $\lambda_{1}$.

The next characterization of $\lambda_{2}$ will be used later. For its proof we refer the reader to [22, Proposition 5].

$\left(\mathrm{p}_{3}\right)$ Write $U:=\left\{u \in X:\|u\|_{p}=1\right\}$ as well as

$$
\begin{gathered}
\Gamma_{1}:=\left\{\gamma \in C^{0}([-1,1], U): \gamma(-1)=-\hat{u}_{1}, \gamma(1)=\hat{u}_{1}\right\}, \\
\Phi(u):=\|\nabla u\|_{p}^{p}+\int_{\partial \Omega} \beta(x)|u(x)|^{p} d \sigma, \quad u \in X .
\end{gathered}
$$

Then

$$
\lambda_{2}=\inf _{\gamma \in \Gamma_{1}} \max _{t \in[-1,1]} \Phi(\gamma(t)) .
$$

Define $U_{C}:=\left\{u \in C^{1}(\bar{\Omega}):\|u\|_{p}=1\right\}$. Evidently, $U_{C}$ turns out to be dense in $U$. Let

$$
\Gamma_{C}:=\left\{\gamma \in C^{0}\left([-1,1], U_{C}\right): \gamma(-1)=-\hat{u}_{1}, \gamma(1)=\hat{u}_{1}\right\}
$$

Lemma 2.1. The set $\Gamma_{C}$ is dense in $\Gamma_{1}$ with respect to the usual norm of $C^{0}([-1,1], X)$.

Proof. Pick any $\gamma \in \Gamma_{1}$. We shall prove that there exists a sequence $\left\{\gamma_{n}\right\} \subseteq \Gamma_{C}$ fulfilling

$$
\lim _{n \rightarrow+\infty} \max _{t \in[-1,1]}\left\|\gamma_{n}(t)-\gamma(t)\right\|=0 .
$$


The multifunction $L_{n}:[-1,1] \rightarrow 2^{C^{1}(\bar{\Omega})}$ defined by

$$
L_{n}(t):= \begin{cases}\left\{-\hat{u}_{1}\right\} & \text { when } t=-1, \\ \left\{u \in C^{1}(\bar{\Omega}):\|u-\gamma(t)\|<1 / n\right\} & \text { if } t \in(-1,1), \\ \left\{\hat{u}_{1}\right\} & \text { when } t=1\end{cases}
$$

takes nonempty convex values and is lower semi-continuous. So, Theorem $3.1^{\prime \prime \prime}$ in [17] provides a continuous selection $l_{n}:[-1,1] \rightarrow C^{1}(\bar{\Omega})$ of $L_{n}$. This entails

$$
\left\|l_{n}(t)-\gamma(t)\right\|<\frac{1}{n} \quad \forall t \in(-1,1), \quad l_{n}(-1)=-\hat{u}_{1}, \quad l_{n}(1)=\hat{u}_{1} .
$$

Consequently,

$$
\lim _{n \rightarrow+\infty}\left\|l_{n}(t)\right\|_{p}=\|\gamma(t)\|_{p}=1
$$

uniformly with respect to $t \in[-1,1]$. For any $n$ large enough we can thus set

$$
\gamma_{n}(t):=\frac{l_{n}(t)}{\left\|l_{n}(t)\right\|_{p}}, \quad t \in[-1,1] .
$$

On account of (2.5) and $\left(\mathrm{p}_{3}\right)$ one has $\gamma_{n} \in \Gamma_{C}$. Moreover,

$$
\begin{array}{r}
\left\|\gamma_{n}(t)-\gamma(t)\right\| \leq\left\|\gamma_{n}(t)-l_{n}(t)\right\|+\left\|l_{n}(t)-\gamma(t)\right\| \\
\quad<\left|1-\left\|l_{n}(t)\right\|_{p}\right| \frac{\left\|l_{n}(t)\right\|}{\left\|l_{n}(t)\right\|_{p}}+\frac{1}{n} \quad \forall t \in[-1,1] .
\end{array}
$$

Recall that $\gamma \in \Gamma_{1}$. Since, by (2.5) again,

$$
\begin{array}{r}
\max _{t \in[-1,1]}\left|1-\left\|l_{n}(t)\right\|_{p}\right|=\max _{t \in[-1,1]}\left|\|\gamma(t)\|_{p}-\left\|l_{n}(t)\right\|_{p}\right| \\
\leq \max _{t \in[-1,1]}\left\|\gamma(t)-l_{n}(t)\right\|_{p} \leq c \max _{t \in[-1,1]}\left\|\gamma(t)-l_{n}(t)\right\| \leq \frac{c}{n}
\end{array}
$$

for some $c>0$, (2.4) immediately follows from (2.5)-(2.7).

Finally, it is known [12, Section 4] that $\left(\mathrm{p}_{4}\right) E\left(\lambda_{n}\right) \subseteq C^{1}(\bar{\Omega})$ for all $n \in \mathbb{N}$.

Let $p:=2$. Through [9, Proposition 3] we also obtain $\left(\mathrm{p}_{5}\right)$ If $u$ lies in $E\left(\lambda_{n}\right)$ and vanishes on a set of positive Lebesgue measure then $u=0$. 
Setting

$$
\bar{H}_{n}:=\oplus_{m=1}^{n} E\left(\lambda_{m}\right), \quad \hat{H}_{n}:=\bar{H}_{n}^{\perp},
$$

each $u \in H^{1}(\Omega)$ can uniquely be written as $u=\bar{u}+\hat{u}$, with $\bar{u} \in \bar{H}_{n}$ and $\hat{u} \in \hat{H}_{n}$, because $H^{1}(\Omega)=\bar{H}_{n} \oplus \hat{H}_{n}$. By orthogonality one has, for every $n \geq 2$,

$$
\begin{aligned}
& \lambda_{n}=\max \left\{\frac{\|\nabla \bar{u}\|_{2}^{2}+\int_{\partial \Omega} \beta \bar{u}^{2} d \sigma}{\|\bar{u}\|_{2}^{2}}: \bar{u} \in \bar{H}_{n}, \bar{u} \neq 0\right\} \\
& =\min \left\{\frac{\|\nabla \hat{u}\|_{2}^{2}+\int_{\partial \Omega} \beta \hat{u}^{2} d \sigma}{\|\hat{u}\|_{2}^{2}}: \hat{u} \in \hat{H}_{n-1}, \hat{u} \neq 0\right\} .
\end{aligned}
$$

A simple argument, based on orthogonality and $\left(\mathrm{p}_{5}\right)$, yields the next result.

Lemma 2.2. Let $n \in \mathbb{N}$ and let $\theta \in L^{\infty}(\Omega) \backslash\left\{\lambda_{n}\right\}$ satisfy $\theta \geq \lambda_{n}$. Then there exists a constant $\bar{c}>0$ such that

$$
\|\nabla \bar{u}\|_{2}^{2}+\int_{\partial \Omega} \beta(x) \bar{u}(x)^{2} d \sigma-\int_{\Omega} \theta(x) \bar{u}(x)^{2} d x \leq-\bar{c}\|\bar{u}\|^{2} \quad \forall \bar{u} \in \bar{H}_{n} .
$$

Let $n \in \mathbb{N}$ and let $\theta \in L^{\infty}(\Omega) \backslash\left\{\lambda_{n+1}\right\}$ satisfy $\theta \leq \lambda_{n+1}$. Then there exists a constant $\hat{c}>0$ such that

$$
\|\nabla \hat{u}\|_{2}^{2}+\int_{\partial \Omega} \beta(x) \hat{u}(x)^{2} d \sigma-\int_{\Omega} \theta(x) \hat{u}(x)^{2} d x \geq \hat{c}\|\hat{u}\|^{2} \quad \forall \hat{u} \in \hat{H}_{n} .
$$

\section{$3 \quad$ Existence results}

To avoid unnecessary technicalities, 'for every $x \in \Omega$ ' will take the place of 'for almost every $x \in \Omega$ ' and the variable $x$ will be omitted when no confusion can arise.

Let $f: \Omega \times \mathbb{R} \rightarrow \mathbb{R}$ be a Carathéodory function such that $f(\cdot, 0)=0$ and let

$$
F(x, \xi):=\int_{0}^{\xi} f(x, t) d t, \quad(x, \xi) \in \Omega \times \mathbb{R} .
$$

We will posit the following assumptions.

$\left(\mathrm{f}_{1}\right)$ To every $\rho>0$ there corresponds $a_{\rho} \in L^{\infty}(\Omega)$ satisfying $\sup _{|t| \leq \rho}|f(x, t)| \leq a_{\rho}(x)$ in $\Omega$.

(f $\left.\mathrm{f}_{2}\right) \limsup _{t \rightarrow \pm \infty} \frac{f(x, t)}{|t|^{p-2} t} \leq a_{0}<\lambda_{1}$ uniformly with respect to $x \in \Omega$. 
$\left(\mathrm{f}_{3}\right)$ There exist $a_{1}, a_{2} \in L^{\infty}(\Omega) \backslash\left\{\lambda_{1}\right\}$ such that $\lambda_{1} \leq a_{1} \leq a_{2}$ and

$$
a_{1}(x) \leq \liminf _{t \rightarrow 0} \frac{f(x, t)}{|t|^{p-2} t} \leq \limsup _{t \rightarrow 0} \frac{f(x, t)}{\mid t^{p-2} t} \leq a_{2}(x) \quad \text { uniformly in } x \in \Omega .
$$

$\left(\mathrm{f}_{4}\right)$ To every $\rho>0$ there corresponds $\mu_{\rho}>0$ such that $t \mapsto f(x, t)+\mu_{\rho}|t|^{p-2} t$ is nondecreasing on $[-\rho, \rho]$ for all $x \in \Omega$.

Remark 3.1. Obviously, both $\left(\mathrm{f}_{3}\right)$ and $\left(\mathrm{f}_{4}\right)$ imply that for each $\rho>0$ we can find $\mu_{\rho}>0$ fulfilling

$$
f(x, t) t+\mu_{\rho}|t|^{p} \geq 0 \quad \forall(x, t) \in \Omega \times[-\rho, \rho] .
$$

Now, recall that $X:=W^{1, p}(\Omega)$. The energy functional $\varphi: X \rightarrow \mathbb{R}$ stemming from Problem (1.1) is

$$
\varphi(u):=\frac{1}{p}\left(\|\nabla u\|_{p}^{p}+\int_{\partial \Omega} \beta(x)|u(x)|^{p} d \sigma\right)-\int_{\Omega} F(x, u(x)) d x, \quad u \in X,
$$

with $F$ given by (3.1). One clearly has $\varphi \in C^{1}(X)$. Moreover, if $\left(\mathrm{f}_{2}\right)$ holds then, fixed any $\hat{a}_{0} \in\left(a_{0}, \lambda_{1}\right)$, there exists $M>0$ such that

$$
\frac{f(x, t)}{|t|^{p-2} t}<\hat{a}_{0}<\lambda_{1}
$$

provided $x \in \Omega$ and $|t| \geq M$. Since $\left(\mathrm{p}_{2}\right)$ entails $t_{1} \hat{u}_{1} \geq M$ for $t_{1}>0$ large enough, inequality (3.3) combined with Remark 2.1 lead to

$$
\int_{\Omega} f(x, \hat{u}) v d x \leq \lambda_{1} \int_{\Omega} \hat{u}^{p-1} d x=\left\langle A_{p}(\hat{u}), v\right\rangle+\int_{\partial \Omega} \beta \hat{u}^{p-1} v d x, \quad v \in X, v \geq 0,
$$

where $\hat{u}:=t_{1} \hat{u}_{1}$.

\subsection{Constant-sign solutions}

Define, provided $x \in \Omega$ and $t, \xi \in \mathbb{R}$,

$$
\begin{gathered}
\hat{g}_{+}(x, t):= \begin{cases}0 & \text { when } t<0, \\
f(x, t)+t^{p-1} & \text { if } 0 \leq t \leq \hat{u}(x), \\
f(x, \hat{t})+\hat{t}^{p-1} & \text { otherwise, }\end{cases} \\
\hat{g}_{-}(x, t):= \begin{cases}f(x,-\hat{t})-\hat{t}^{p-1} & \text { when } t<-\hat{u}(x), \\
f(x, t)+|t|^{p-2} t & \text { if }-\hat{u}(x) \leq t \leq 0, \\
0 & \text { otherwise, }\end{cases}
\end{gathered}
$$


as well as

$$
\hat{G}_{ \pm}(x, \xi):=\int_{0}^{\xi} \hat{g}_{ \pm}(x, t) d t .
$$

It is evident that the corresponding truncated functionals

$$
\hat{\psi}_{ \pm}(u):=\frac{1}{p}\left(\|u\|^{p}+\int_{\partial \Omega} \beta(x)|u(x)|^{p} d \sigma\right)-\int_{\Omega} \hat{G}_{ \pm}(x, u(x)) d x, \quad u \in X,
$$

belong to $C^{1}(X)$ also.

Theorem 3.1. Under hypotheses $\left(\mathrm{f}_{1}\right)-\left(\mathrm{f}_{3}\right)$, Problem (1.1) admits at least two constantsign solutions $u_{0} \in[0, \hat{u}] \cap \operatorname{int}\left(C_{+}\right)$and $v_{0} \in[-\hat{u}, 0] \cap\left(-\operatorname{int}\left(C_{+}\right)\right)$. If, moreover, $\left(\mathrm{f}_{4}\right)$ holds then $u_{0}, v_{0}$ are local minimizers for $\varphi$.

Proof. The space $X$ compactly embeds in $L^{p}(\Omega)$ while the Nemitskii operator $N_{\hat{g}_{+}}$turns out to be continuous on $L^{p}(\Omega)$. Thus, a standard argument ensures that $\hat{\psi}_{+}$is weakly sequentially lower semi-continuous. Since, on account of (3.5), it is coercive, we have

$$
\inf _{u \in X} \hat{\psi}_{+}(u)=\hat{\psi}_{+}\left(u_{0}\right)
$$

for some $u_{0} \in X$. Fix $\varepsilon>0$. Assumption ( $\left.\mathrm{f}_{3}\right)$ yields $\delta>0$ small such that

$$
F(x, \xi) \geq \frac{a_{1}(x)-\varepsilon}{p}|\xi|^{p} \quad \forall(x, \xi) \in \Omega \times[-\delta, \delta] .
$$

If $\tau \in\left(0, t_{1}\right)$ complies with $\tau \hat{u}_{1} \leq \delta$ then, by (3.5), the choice of $\tau$, the above inequality, and Remark 2.1,

$$
\begin{array}{r}
\hat{\psi}_{+}\left(\tau \hat{u}_{1}\right) \leq \frac{\tau^{p}}{p}\left(\left\|\nabla \hat{u}_{1}\right\|_{p}^{p}+\int_{\partial \Omega} \beta \hat{u}_{1}^{p} d \sigma-\int_{\Omega}\left(a_{1}-\varepsilon\right) \hat{u}_{1}^{p} d x\right) \\
=\frac{\tau^{p}}{p}\left(\lambda_{1} \int_{\Omega} \hat{u}_{1}^{p} d x-\int_{\Omega}\left(a_{1}-\varepsilon\right) \hat{u}_{1}^{p} d x\right)=\frac{\tau^{p}}{p}\left(\int_{\Omega}\left(\lambda_{1}-a_{1}\right) \hat{u}_{1}^{p} d x+\varepsilon\right)<0
\end{array}
$$

as soon as $\varepsilon<\int_{\Omega}\left(\lambda_{1}-a_{1}\right) \hat{u}_{1}^{p} d x$. Hence,

$$
\hat{\psi}_{+}\left(u_{0}\right)<0=\hat{\psi}_{+}(0),
$$

which clearly means $u_{0} \neq 0$. Now, through (3.6) we get $\hat{\psi}_{+}^{\prime}\left(u_{0}\right)=0$, namely

$$
\left\langle A_{p}\left(u_{0}\right)+\left|u_{0}\right|^{p-2} u_{0}, v\right\rangle+\int_{\partial \Omega} \beta\left|u_{0}\right|^{p-2} u_{0} v d \sigma=\left\langle N_{\hat{g}_{+}}\left(u_{0}\right), v\right\rangle, \quad v \in X .
$$


Choosing $v:=-u_{0}^{-}$in (3.7) leads to $\left\|\nabla u_{0}^{-}\right\|_{p}^{p}+\left\|u_{0}^{-}\right\|_{p}^{p} \leq 0$, and $u_{0} \geq 0$. Next, pick $v:=\left(u_{0}-\hat{u}\right)^{+}$. From (3.7), besides (3.4), it follows

$$
\begin{array}{r}
\left\langle A_{p}\left(u_{0}\right),\left(u_{0}-\hat{u}\right)^{+}\right\rangle+\int_{\Omega} u_{0}^{p-1}\left(u_{0}-\hat{u}\right)^{+} d x+\int_{\partial \Omega} \beta u_{0}^{p-1}\left(u_{0}-\hat{u}\right)^{+} d \sigma \\
=\int_{\Omega}\left(f(x, \hat{u})+\hat{u}^{p-1}\right)\left(u_{0}-\hat{u}\right)^{+} d x \\
\leq\left\langle A_{p}(\hat{u}),\left(u_{0}-\hat{u}\right)^{+}\right\rangle+\int_{\partial \Omega} \beta \hat{u}^{p-1}\left(u_{0}-\hat{u}\right)^{+} d \sigma+\int_{\Omega} \hat{u}^{p-1}\left(u_{0}-\hat{u}\right)^{+} d x,
\end{array}
$$

that is

$$
\left\langle A_{p}\left(u_{0}\right)-A_{p}(\hat{u}),\left(u_{0}-\hat{u}\right)^{+}\right\rangle+\int_{\partial \Omega} \beta\left(u_{0}^{p-1}-\hat{u}^{p-1}\right)\left(u_{0}-\hat{u}\right)^{+} d \sigma+\int_{\Omega}\left(u_{0}^{p-1}-\hat{u}^{p-1}\right)\left(u_{0}-\hat{u}\right)^{+} d x \leq 0 .
$$

Consequently, $u_{0} \leq \hat{u}$. Now, (3.7) becomes

$$
\left\langle A_{p}\left(u_{0}\right), v\right\rangle+\int_{\partial \Omega} \beta u_{0}^{p-1} v d \sigma=\int_{\Omega} f\left(x, u_{0}\right) v d x \quad \forall v \in X
$$

whence, on account of Remark 2.1,

$$
-\Delta_{p} u_{0}=f\left(x, u_{0}\right) \quad \text { in } \quad \Omega, \quad \frac{\partial u}{\partial n_{p}}+\beta(x)\left|u_{0}\right|^{p-2} u_{0}=0 \quad \text { on } \quad \partial \Omega .
$$

Standard regularity arguments ensure that $u_{0} \in C_{+} \backslash\{0\}$. Let $\rho:=\|\hat{u}\|_{\infty} \geq\left\|u_{0}\right\|_{\infty}$. Due to Remark 3.1 one has

$$
-\Delta_{p} u_{0}(x)+\mu_{\rho} u_{0}(x)^{p-1}=f\left(x, u_{0}(x)\right)+\mu_{\rho} u_{0}(x)^{p-1} \geq 0 \quad \text { a.e. in } \Omega .
$$

Therefore, by [25, Theorem 5], $u_{0} \in \operatorname{int}\left(C_{+}\right)$and thus $u_{0} \in[0, \hat{u}] \cap \operatorname{int}\left(C_{+}\right)$, as desired. Define $u_{\delta}:=u_{0}+\delta$, where $\delta>0$. Since

$$
-\Delta_{p} u_{\delta}(x)+\mu_{\rho} u_{\delta}(x)^{p-1} \leq-\Delta_{p} u_{0}(x)+\mu_{\rho} u_{0}(x)^{p-1}+o(\delta)=f\left(x, u_{0}(x)\right)+\mu_{\rho} u_{0}(x)^{p-1}+o(\delta),
$$

exploiting $\left(\mathrm{f}_{4}\right)$ and $(\underline{3.3})$ we obtain

$$
\begin{array}{r}
-\Delta_{p} u_{\delta}(x)+\mu_{\rho} u_{\delta}(x)^{p-1} \leq f(x, \hat{u}(x))+\mu_{\rho} \hat{u}(x)^{p-1}+o(\delta) \\
<\left(\hat{a}_{0}+\mu_{\rho}\right) \hat{u}(x)^{p-1}+o(\delta) \leq\left(\lambda_{1}+\mu_{\rho}\right) \hat{u}(x)^{p-1}=-\Delta_{p} \hat{u}(x)+\mu_{\rho} \hat{u}(x)^{p-1}
\end{array}
$$

for any $\delta>0$ small enough, because

$$
\left(\lambda_{1}-\hat{a}_{0}\right) \inf _{x \in \Omega} \hat{u}(x)^{p-1}>0
$$


cf. (3.3) as well as $\left(\mathrm{p}_{2}\right)$. Theorem 5 of [25] gives $u_{\delta} \leq \hat{u}$, whence

$$
u_{0} \in \operatorname{int}_{C^{1}(\bar{\Omega})}([0, \hat{u}]) .
$$

Observe next that $\left.\varphi\right|_{[0, \hat{u}]}=\left.\hat{\psi}_{+}\right|_{[0, \hat{u}]}$ thanks to (3.5) . So, by (3.8) and (3.6), the function $u_{0}$ is a $C^{1}(\bar{\Omega})$-local minimizer for $\varphi$. Finally, [22, Proposition 3] guarantees that the same holds putting $X$ in place of $C^{1}(\bar{\Omega})$.

A similar argument produces $v_{0} \in[-\hat{u}, 0] \cap\left(-\operatorname{int}\left(C_{+}\right)\right)$with the asserted properties.

Remark 3.2. The upper bound at zero requested by $\left(\mathrm{f}_{3}\right)$ for $t \mapsto f(x, t) /|t|^{p-2} t$ has not been used to find constant-sign solutions.

The next result looks like [13, Theorem 3.3]; see also [22, Proposition 8]. So, we will only sketch its proof.

Theorem 3.2. Let $\left(\mathrm{f}_{1}\right)-\left(\mathrm{f}_{3}\right)$ be satisfied. Then (1.1) possesses the smallest (resp., the biggest) nontrivial solution $u_{*}$ in $[0, \hat{u}]$ (resp., $v_{*}$ in $[-\hat{u}, 0]$ ). Further, $-v_{*}, u_{*} \in \operatorname{int}\left(C_{+}\right)$.

Proof. Define $\Sigma_{+}:=\{u \in X \backslash\{0\}: u$ solves (1.1) and $0 \leq u \leq \hat{u}\}$. Due to Theorem 3.1 one has $\Sigma_{+} \neq \emptyset$. Actually, $\Sigma_{+} \subseteq \operatorname{int}\left(C_{+}\right)$. The same arguments employed in establishing [2, Proposition 8] show here that

1) $\Sigma_{+}$is downward directed, and

$2) \inf \Sigma_{+}=\inf _{n \in \mathbb{N}} u_{n}=u_{*}$ for some $\left\{u_{n}\right\} \subseteq \Sigma_{+}, u_{*} \in X$ fulfilling $u_{n} \rightarrow u_{*}$ in $X$ and $u_{n}(x) \rightarrow u_{*}(x)$ a.e. in $\Omega$.

Hence, $u_{*}$ turns out to be a solution of (1.1) lying in $[0, \hat{u}]$. It remains to verify that $u_{*} \neq 0$. Suppose on the contrary $u_{*}=0$. Reasoning exactly as in the proof of [1, Proposition 14] we obtain $\alpha \in L^{\infty}(\Omega)$ and $w \in \operatorname{int}\left(C_{+}\right)$with the properties below.

3) $a_{1} \leq \alpha \leq a_{2}$

4) $-\Delta_{p} w(x)=\alpha(x)|w(x)|^{p-2} w(x)$ a.e. in $\Omega, \frac{\partial w}{\partial n_{p}}+\beta(x)|w|^{p-2} w=0$ on $\partial \Omega$.

Let $v \in \operatorname{int}\left(C_{+}\right)$. Gathering [3, Theorem 1.1] and [10, Theorem 2.4.54] together produce

$$
\begin{array}{r}
0 \leq \int_{\Omega}\left(|\nabla v|^{p}-\nabla\left(\frac{v^{p}}{w^{p-1}}\right) \cdot|\nabla w|^{p-2} \nabla w\right) d x \\
=\int_{\Omega}\left(|\nabla v|^{p}-\frac{v^{p}}{w^{p-1}}\left(-\Delta_{p} w\right)\right) d x-\int_{\partial \Omega} \frac{\partial u}{\partial n_{p}} \frac{v^{p}}{w^{p-1}} d \sigma \\
=\int_{\Omega}\left(|\nabla v|^{p}-\alpha v^{p}\right) d x+\int_{\partial \Omega} \beta v^{p} d \sigma,
\end{array}
$$


where 4) has been used. If $v=\hat{u}_{1}$ then, by 3$)$ and $\left(\mathrm{f}_{3}\right)$,

$$
0 \leq \int_{\Omega}\left(\lambda_{1}-\alpha(x)\right) \hat{u}_{1}(x)^{p} d x \leq \int_{\Omega}\left(\lambda_{1}-a_{1}(x)\right) \hat{u}_{1}(x)^{p} d x<0,
$$

which is impossible. Therefore, $u_{*} \in \Sigma_{+}$, and the conclusion follows. A similar argument applies to get $v_{*}$.

\subsection{Nodal solutions}

Define, for every $x \in \Omega$ and $t, \xi \in \mathbb{R}$,

$$
\begin{aligned}
& \hat{f}(x, t):= \begin{cases}f\left(x, v_{*}(x)\right)+\left|v_{*}(x)\right|^{p-2} v_{*}(x) & \text { when } t<v_{*}(x), \\
f(x, t)+|t|^{p-2} t & \text { if } v_{*}(x) \leq t \leq u_{*}(x), \\
f\left(x, u_{*}(x)\right)+u_{*}(x)^{p-1} & \text { when } t>u_{*}(x),\end{cases} \\
& \hat{f}_{ \pm}(x, t):=\hat{f}\left(x, t^{ \pm}\right),
\end{aligned}
$$

as well as

$$
\hat{F}(x, \xi):=\int_{0}^{\xi} \hat{f}(x, t) d t, \quad \hat{F}_{ \pm}(x, \xi):=\int_{0}^{\xi} \hat{f}_{ \pm}(x, t) d t .
$$

It is evident that the corresponding truncated functionals

$$
\begin{aligned}
\hat{\varphi}(u) & :=\frac{1}{p}\left(\|u\|^{p}+\int_{\partial \Omega} \beta(x)|u(x)|^{p-1} d \sigma\right)-\int_{\Omega} \hat{F}(x, u(x)) d x, \quad u \in X, \\
\hat{\varphi}_{ \pm}(u) & :=\frac{1}{p}\left(\|u\|^{p}+\int_{\partial \Omega} \beta(x)|u(x)|^{p-1} d \sigma\right)-\int_{\Omega} \hat{F}_{ \pm}(x, u(x)) d x, \quad u \in X,
\end{aligned}
$$

belong to $C^{1}(X)$.

Lemma 3.1. Under hypotheses $\left(\mathrm{f}_{1}\right)-\left(\mathrm{f}_{3}\right)$ one has

$$
K(\hat{\varphi}) \subseteq\left[v_{*}, u_{*}\right], \quad K\left(\hat{\varphi}_{-}\right)=\left\{0, v_{*}\right\}, \quad K\left(\hat{\varphi}_{+}\right)=\left\{0, u_{*}\right\} .
$$

Proof. If $u \in K(\hat{\varphi})$ then

$$
\left\langle A_{p}(u)+|u|^{p-2} u, v\right\rangle+\int_{\partial \Omega} \beta|u|^{p-2} u v d \sigma=\left\langle N_{\hat{f}}(u), v\right\rangle \quad \forall v \in X .
$$

Letting $v:=\left(u-u_{*}\right)^{+}$yields

$$
\begin{aligned}
\left\langle A_{p}(u),\left(u-u_{*}\right)^{+}\right\rangle+\int_{\Omega} u^{p-1}(u & \left.-u_{*}\right)^{+} d x+\int_{\partial \Omega} \beta|u|^{p-1}\left(u-u_{*}\right)^{+} d \sigma \\
& =\int_{\Omega}\left(f\left(x, u_{*}\right)+u_{*}^{p-1}\right)\left(u-u_{*}\right)^{+} d x .
\end{aligned}
$$


Since, by Theorem 3.2 , the function $u_{*}$ solves (1.1), this results in

$$
\left\langle A_{p}(u)-A_{p}\left(u_{*}\right),\left(u-u_{*}\right)^{+}\right\rangle+\int_{\Omega}\left(u^{p-1}-u_{*}^{p-1}\right)\left(u-u_{*}\right)^{+} d x+\int_{\partial \Omega} \beta\left(u^{p-1}-u_{*}^{p-1}\right)\left(u-u_{*}\right)^{+} d \sigma=0 .
$$

Therefore, $m\left(\left\{x \in \Omega: u(x)>u_{*}(x)\right\}\right)=0$, whence $u \leq u_{*}$. An analogous reasoning provides $u \geq v_{*}$, and the first inclusion holds.

As before, we obtain $K\left(\hat{\varphi}_{-}\right) \subseteq\left[v_{*}, 0\right]$, while the extremality of $v_{*}$ (see Theorem 3.2) forces $K\left(\hat{\varphi}_{-}\right)=\left\{v_{*}, 0\right\}$. The remaining proof is similar.

Lemma 3.2. Let $\left(\mathrm{f}_{1}\right)-\left(\mathrm{f}_{4}\right)$ be satisfied. Then $u_{*}, v_{*}$ are local minimizers for $\hat{\varphi}$.

Proof. The space $X$ compactly embeds in $L^{p}(\Omega)$ while the Nemitskii operator $N_{\hat{f}_{+}}$turns out to be continuous on $L^{p}(\Omega)$. Thus, a standard argument ensures that $\hat{\varphi}_{+}$is weakly sequentially lower semi-continuous. Since, on account of (3.9), it is coercive, we have

$$
\inf _{u \in X} \hat{\varphi}_{+}(u)=\hat{\varphi}_{+}\left(u_{0}\right)
$$

for some $u_{0} \in X$. Reasoning as in the proof of Theorem 3.1 produces $\hat{\varphi}_{+}\left(u_{0}\right)<0$, i.e., $u_{0} \neq 0$. Hence, by (3.10) and Lemma 3.1, $u_{0}=u_{*} \in \operatorname{int}\left(C_{+}\right)$. Since $\left.\hat{\varphi}\right|_{C_{+}}=\left.\hat{\varphi}_{+}\right|_{C_{+}}$, the function $u_{0}$ turns out to be a $C^{1}(\bar{\Omega})$-local minimizer for $\hat{\varphi}$. Now, Proposition 3 in [22] guarantees that the same holds true with $X$ in place of $C^{1}(\bar{\Omega})$. A similar argument applies to $v_{*}$.

Theorem 3.3. Under hypotheses $\left(\mathrm{f}_{1}\right)-\left(\mathrm{f}_{4}\right)$, with $\underset{x \in \Omega}{\operatorname{ess} \inf } a_{1}(x)>\lambda_{2}$, Problem (1.1) possesses a nodal solution $u_{1} \in\left[v_{*}, u_{*}\right] \cap C^{1}(\bar{\Omega})$.

Proof. By Theorem 3.2 and Lemma 3.1, we may assume $K(\hat{\varphi})$ finite. Let $\hat{\varphi}\left(v_{*}\right) \leq \hat{\varphi}\left(u_{*}\right)$ (the other case is analogous). Without loss of generality, the local minimizer $u_{*}$ for $\hat{\varphi}$ (cf. Lemma 3.2) can be supposed proper. Thus, there exists $\rho \in\left(0,\left\|u_{*}-v_{*}\right\|\right)$ such that

$$
\hat{\varphi}\left(u_{*}\right)<c_{\rho}:=\inf _{u \in \partial B_{\rho}\left(u_{*}\right)} \hat{\varphi}(u) .
$$

Moreover, $\hat{\varphi}$ fulfils Condition (PS) because, due to (3.9), it is coercive; see Proposition 2.1. So, the Mountain Pass Theorem yields a point $u_{1} \in X$ complying with $\hat{\varphi}^{\prime}\left(u_{1}\right)=0$ and

$$
c_{\rho} \leq \hat{\varphi}\left(u_{1}\right)=\inf _{\gamma \in \Gamma} \max _{t \in[0,1]} \hat{\varphi}(\gamma(t))
$$

where

$$
\Gamma:=\left\{\gamma \in C^{0}([0,1], X): \gamma(0)=v_{*}, \gamma(1)=u_{*}\right\}
$$


Obviously, $u_{1}$ solves (1.1). Through (3.11)-(3.12), besides Lemma 3.1, we get

$$
u_{1} \in\left[v_{*}, u_{*}\right] \backslash\left\{v_{*}, u_{*}\right\},
$$

while standard regularity arguments yield $u_{1} \in C^{1}(\bar{\Omega})$. The proof is thus completed once one verifies that $u_{1} \neq 0$. This will follow from the inequality

$$
\hat{\varphi}\left(u_{1}\right)<0
$$

which, in view of (3.12), can be shown by constructing a path $\tilde{\gamma} \in \Gamma$ such that

$$
\hat{\varphi}(\tilde{\gamma}(t))<0 \quad \forall t \in[0,1] \text {. }
$$

By $\left(\mathrm{f}_{3}\right)$ to every $\eta>0$ there corresponds $\delta>0$ such that

$$
F(x, \xi) \geq \frac{a_{1}(x)-\eta}{p}|\xi|^{p}, \quad(x, z) \in \Omega \times[-\delta, \delta] .
$$

Combining $\left(\mathrm{p}_{4}\right)$ with Lemma 2.1 entails

$$
\max _{t \in[-1,1]} \Phi\left(\gamma_{\eta}(t)\right)<\lambda_{2}+\eta
$$

for appropriate $\gamma_{\eta} \in \Gamma_{C}$. Since $\gamma_{\eta}([-1,1])$ is compact in $C^{1}(\bar{\Omega})$ and $-v_{*}, u_{*} \in \operatorname{int}\left(C_{+}\right)$we can find $\varepsilon>0$ so small that

$$
v_{*}(x) \leq \varepsilon \gamma_{\eta}(t)(x) \leq u_{*}(x), \quad\left|\varepsilon \gamma_{\eta}(t)(x)\right| \leq \delta
$$

whenever $x \in \Omega, t \in[-1,1]$. Thanks to (3.15)-(3.16) one has

$$
\begin{array}{r}
\hat{\varphi}\left(\varepsilon \gamma_{\eta}(t)\right)=\frac{\varepsilon^{p}}{p}\left(\left\|\gamma_{\eta}(t)\right\|^{p}+\int_{\partial \Omega} \beta\left|\gamma_{\eta}(t)\right|^{p} d \sigma\right)-\int_{\Omega} \hat{F}\left(x, \varepsilon \gamma_{\eta}(t)(x)\right) d x \\
<\frac{\varepsilon^{p}}{p}\left(\Phi\left(\gamma_{\eta}(t)\right)+\int_{\Omega}\left(\eta-a_{1}\right)\left|\gamma_{\eta}(t)\right|^{p} d x\right)<\frac{\varepsilon^{p}}{p}\left(\lambda_{2}+2 \eta-\underset{x \in \Omega}{\operatorname{essinf}} a_{1}(x)\right)<0
\end{array}
$$

provided $\eta<\frac{1}{2}\left(\underset{x \in \Omega}{\operatorname{ess} \inf } a_{1}(x)-\lambda_{2}\right)$, because $\gamma_{\eta}(t) \in U_{C}$. Consequently,

$$
\left.\hat{\varphi}\right|_{\varepsilon \gamma_{\eta}([-1,1])}<0
$$

Next, write $a:=\hat{\varphi}_{+}\left(u_{*}\right)$. From the proof of Lemma 3.2 it follows $a<0$. We may suppose

$$
K\left(\hat{\varphi}_{+}\right)=\left\{0, u_{*}\right\},
$$


otherwise the conclusion is straightforward. Hence, no critical value of $\hat{\varphi}_{+}$lies in $(a, 0)$ while

$$
K_{a}\left(\hat{\varphi}_{+}\right)=\left\{u_{*}\right\} .
$$

Due to the second deformation lemma [10, Theorem 5.1.33], there exists a continuous function $h:[0,1] \times\left(\hat{\varphi}_{+}^{0} \backslash\{0\}\right) \rightarrow \hat{\varphi}_{+}^{0}$ satisfying

$$
h(0, u)=u, \quad h(1, u)=u_{*}, \quad \text { and } \quad \hat{\varphi}_{+}(h(t, u)) \leq \hat{\varphi}_{+}(u)
$$

for all $(t, u) \in[0,1] \times\left(\hat{\varphi}_{+}^{0} \backslash\{0\}\right)$. Let $\gamma_{+}(t):=h\left(t, \varepsilon \hat{u}_{0}\right)^{+}, t \in[0,1]$. Then $\gamma_{+}(0)=\varepsilon \hat{u}_{0}$, $\gamma_{+}(1)=u_{*}$, as well as

$$
\hat{\varphi}\left(\gamma_{+}(t)\right)=\hat{\varphi}_{+}\left(\gamma_{+}(t)\right) \leq \hat{\varphi}_{+}\left(h\left(t, \varepsilon \hat{u}_{0}\right)\right) \leq \hat{\varphi}_{+}\left(\varepsilon \hat{u}_{0}\right)=\hat{\varphi}\left(\varepsilon \gamma_{\eta}(1)\right)<0
$$

cf. (3.17). In a similar way, but with $\hat{\varphi}_{+}$replaced by $\hat{\varphi}_{-}$, we can construct a continuous function $\gamma_{-}:[0,1] \rightarrow X$ such that $\gamma_{-}(0)=v_{*}, \gamma_{-}(1)=-\varepsilon \hat{u}_{0}$, and

$$
\varphi\left(\gamma_{-}(t)\right)<0 \quad \forall t \in[0,1]
$$

Concatenating $\gamma_{-}, \varepsilon \gamma_{\eta}$, and $\gamma_{+}$one obtains a path $\tilde{\gamma} \in \Gamma$ which, in view of (3.17)-(3.19), fulfils (3.14).

The next multiplicity result directly stems from Theorems 3.13 .3 .

Theorem 3.4. Let $\left(\mathrm{f}_{1}\right)-\left(\mathrm{f}_{4}\right)$ be satisfied and let $\underset{x \in \Omega}{\operatorname{essinf}} a_{1}(x)>\lambda_{2}$. Then (1.1) admits at least three nontrivial solutions: $u_{0} \in \operatorname{int}\left(C_{+}\right), v_{0} \in-\operatorname{int}\left(C_{+}\right)$, and $u_{1} \in\left[v_{0}, u_{0}\right] \cap C^{1}(\bar{\Omega})$ nodal.

An immediate application of this result produces both constant-sign and nodal solutions to the problem

$$
\begin{cases}-\Delta_{p} u=\lambda|u|^{p-2} u-g(x, u) & \text { in } \Omega \\ \frac{\partial u}{\partial n_{p}}+\beta(x)|u|^{p-2} u=0 & \text { on } \partial \Omega\end{cases}
$$

where $\lambda>0$ while $g: \Omega \times \mathbb{R} \rightarrow \mathbb{R}$ denotes a Carathéodory function such that $g(\cdot, 0)=0$. Under Dirichlet boundary conditions, the above equation has been widely investigated; see for instance [21, 4, 19] and the references given there.

Theorem 3.5. Assume that $\lambda>\lambda_{2}$. If, moreover, $\left(\mathrm{g}_{1}\right)$ to every $\rho>0$ there corresponds $b_{\rho} \in L^{\infty}(\Omega)$ satisfying $\sup _{|t| \leq \rho}|g(x, t)| \leq b_{\rho}(x)$ in $\Omega$, 
$\left(\mathrm{g}_{2}\right) \liminf _{t \rightarrow \pm \infty} \frac{g(x, t)}{|t|^{p-2} t} \geq b_{0}>\lambda-\lambda_{1}$ uniformly with respect to $x \in \Omega$,

$\left(\mathrm{g}_{3}\right) b_{1} \leq \liminf _{t \rightarrow 0} \frac{g(x, t)}{|t|^{p-2} t} \leq \limsup _{t \rightarrow 0} \frac{g(x, t)}{|t|^{p-2} t} \leq b_{2}<\lambda-\lambda_{1}$ uniformly in $x \in \Omega$, as well as

$\left(\mathrm{g}_{4}\right)$ for every $\rho>0$ there exists $\mu_{\rho}>\lambda$ such that $t \mapsto \mu_{\rho}|t|^{p-2} t-g(x, t)$ is nondecreasing on $[-\rho, \rho]$ whatever $x \in \Omega$,

then (3.20) possesses at least three nontrivial solutions: $u_{0} \in \operatorname{int}\left(C_{+}\right), v_{0} \in-\operatorname{int}\left(C_{+}\right)$, and $u_{1} \in\left[v_{0}, u_{0}\right] \cap C^{1}(\bar{\Omega})$ nodal.

Conditions $\left(\mathrm{g}_{2}\right)-\left(\mathrm{g}_{3}\right)$ above are much more general than the corresponding ones of $[22$, Theorem 12] but $\left(\mathrm{g}_{4}\right)$ does not appear in that result. A similar comment holds true for [11, Theorem 3.1], where $\beta \equiv 0$ and sub-critical behavior for $t \mapsto g(x, t)$ is taken on. Finally, the $\beta \equiv 0$ version of Theorem 3.5] and [5, Theorem 4.1] are mutually independent.

\section{The semilinear case}

Suppose $f: \Omega \times \mathbb{R} \rightarrow \mathbb{R}$ is a function such that $f(\cdot, 0)=0$ and $f(x, \cdot)$ belongs to $C^{1}(\mathbb{R})$ for every $x \in \Omega$, while $f(\cdot, t)$ and $f_{t}^{\prime}(\cdot, t)$ are measurable for all $t \in \mathbb{R}$. Let $F$ be given by (3.1). We will make the following assumptions.

$\left(f_{5}\right)\left|f_{t}^{\prime}(x, t)\right| \leq a_{3}\left(1+|t|^{r-2}\right)$ in $\Omega \times \mathbb{R}$, where $2 \leq r<2^{*}$.

$\left(\mathrm{f}_{6}\right) f_{t}^{\prime}(x, 0)=\lim _{t \rightarrow 0} \frac{f(x, t)}{t}$ uniformly with respect to $x \in \Omega$. Moreover, there exists $m \geq 2$ such that $\lambda_{m} \leq f_{t}^{\prime}(\cdot, 0) \leq \lambda_{m+1}, f_{t}^{\prime}(\cdot, 0) \neq \lambda_{m}$, and

$$
F(x, \xi) \leq \frac{\lambda_{m+1}}{2} \xi^{2} \quad \forall(x, \xi) \in \Omega \times \mathbb{R} .
$$

$\left(\mathrm{f}_{7}\right)$ If $m=2$ then $\lambda_{2}<a_{4} \leq f_{t}^{\prime}(x, 0)$ uniformly in $x \in \Omega$.

It should be noted that $\left(f_{5}\right)$ implies both $\left(f_{1}\right)$ and $\left(f_{4}\right)$ written for $p=2$, while $\left(f_{6}\right)$ forces $\left(\mathrm{f}_{3}\right)$ with $p=2$. Consider the semi-linear problem

$$
\begin{cases}-\Delta u=f(x, u) & \text { in } \Omega, \\ \frac{\partial u}{\partial n}+\beta(x) u=0 & \text { on } \partial \Omega,\end{cases}
$$


where $\frac{\partial u}{\partial n}:=\nabla u \cdot n$; see [18, Remark 1.40]. If $X:=H^{1}(\Omega)$ then the energy functional $\varphi: X \rightarrow \mathbb{R}$ stemming from (4.1) is

$$
\varphi(u):=\frac{1}{2}\left(\|\nabla u\|_{2}^{2}+\int_{\partial \Omega} \beta(x) u(x)^{2} d \sigma\right)-\int_{\Omega} F(x, u(x)) d x, \quad u \in X .
$$

Obviously, $\varphi \in C^{2}(X)$ and one has

$$
\left\langle\varphi^{\prime \prime}(u)(v), w\right\rangle=\int_{\Omega} \nabla v \cdot \nabla w d x+\int_{\partial \Omega} \beta v w d \sigma-\int_{\Omega} f_{t}^{\prime}(x, u) v w d x \quad \forall u, v, w \in X .
$$

Lemma 4.1. Let $\left(\mathrm{f}_{5}\right)-\left(\mathrm{f}_{6}\right)$ be satisfied. Then $C_{q}(\varphi, 0)=\delta_{q, d_{m}} \mathbb{Z}$ for all $q \in \mathbb{N}_{0}$, where $d_{m}:=\operatorname{dim}\left(\bar{H}_{m}\right)$.

Proof. Suppose $f_{t}^{\prime}(\cdot, 0) \neq \lambda_{m+1}$. By $\left(\mathrm{f}_{6}\right)$, Lemma 2.2 can be applied with $\theta(x):=f_{t}^{\prime}(x, 0)$. So, $u=0$ is a non-degenerate critical point of $\varphi$ having Morse index $d_{m}$, and the conclusion follows from (2.2). Let now $f_{t}^{\prime}(\cdot, 0)=\lambda_{m+1}$. Thanks to $\left(\mathrm{f}_{6}\right)$ again, for every $\varepsilon>0$ there exists $\delta>0$ such that

$$
F(x, \xi) \geq \frac{1}{2}\left(f_{t}^{\prime}(x, 0)-\varepsilon\right)|\xi|^{2} \quad \text { in } \quad \Omega \times[-\delta, \delta] .
$$

Since $\bar{H}_{m}$ is finite dimensional, we can find $\rho>0$ fulfilling

$$
u \in \bar{H}_{m} \cap B_{\rho}(0) \Longrightarrow|u(x)| \leq \delta \quad \forall x \in \Omega .
$$

Through (4.4) and Lemma 2.2 this entails

$$
\begin{aligned}
\varphi(u) \leq \frac{1}{2}\left(\|\nabla u\|_{2}^{2}\right. & \left.+\int_{\partial \Omega} \beta u^{2} d \sigma-\int_{\Omega} f_{t}^{\prime}(x, 0) u^{2} d x\right)+\frac{\varepsilon}{2}\|u\|^{2} \\
& \leq \frac{1}{2}(-\bar{c}+\varepsilon)\|u\|^{2} \leq 0, \quad u \in \bar{H}_{m} \cap B_{\rho}(0)
\end{aligned}
$$

whenever $\varepsilon<\bar{c}$. Combining $\left(\mathrm{f}_{6}\right)$ with $(2.8)$ we obtain

$$
\varphi(u) \geq \frac{1}{2}\left(\|\nabla u\|_{2}^{2}+\int_{\partial \Omega} \beta u^{2} d \sigma-\lambda_{m+1}\|u\|_{2}^{2}\right) \geq 0, \quad u \in \hat{H}_{m} \cap B \rho(0) .
$$

Now, [24, Proposition 2.3] directly yields the conclusion.

Theorem 4.1. Under assumptions $\left(\mathrm{f}_{2}\right)$ and $\left(\mathrm{f}_{5}\right)-\left(\mathrm{f}_{7}\right)$, Problem (4.1) admits at least four solutions: $u_{0} \in \operatorname{int}\left(C_{+}\right), v_{0} \in-\operatorname{int}\left(C_{+}\right)$, and $u_{1}, v_{1} \in \operatorname{int}_{C^{1}(\bar{\Omega})}\left(\left[v_{0}, u_{0}\right]\right)$ nodal. 
Proof. The existence of $u_{0}, v_{0}, u_{1}$ comes from Theorem 3.4. Bearing in mind Theorem 3.2 and Lemma 3.2, we may suppose $u_{0}, v_{0}$ extremal constant-sign solutions to (4.1), i.e., $u_{0}=u_{*}, v_{0}=v_{*}$, as well as local minimizers for $\hat{\varphi}$. Thus,

$$
C_{q}\left(\hat{\varphi}, u_{0}\right)=C_{q}\left(\hat{\varphi}, v_{0}\right)=\delta_{q, 0} \mathbb{Z} \quad \forall q \in \mathbb{N}_{0} ;
$$

see [18, Example 6.45]. Let us next verify that $u_{1} \in \operatorname{int}_{C^{1}(\bar{\Omega})}\left(\left[v_{0}, u_{0}\right]\right)$. Put

$$
\rho:=\max \left\{\left\|u_{0}\right\|_{\infty},\left\|v_{0}\right\|_{\infty}\right\} .
$$

If $\mu_{\rho}$ is as in $\left(\mathrm{f}_{4}\right)$ then

$$
-\Delta\left(u_{0}-u_{1}\right)+\mu_{\rho}\left(u_{0}-u_{1}\right)=\left[f\left(x, u_{0}\right)+\mu_{\rho} u_{0}\right]-\left[f\left(x, u_{1}\right)+\mu_{\rho} u_{1}\right] \geq 0
$$

because $u_{1} \leq u_{0}$. So, by [25, Theorem 5], $u_{0}-u_{1} \in \operatorname{int}\left(C_{+}\right)$. Likewise, $u_{1}-v_{0} \in \operatorname{int}\left(C_{+}\right)$, and the assertion follows.

The proof of Theorem 3.3 ensures that $u_{1}$ is a Mountain Pass type critical point for $\hat{\varphi}$. Thanks to (4.3), Corollary 6.102 in [18] gives

$$
C_{q}\left(\hat{\varphi}, u_{1}\right)=\delta_{q, 1} \mathbb{Z}, \quad q \in \mathbb{N}_{0}
$$

From $\left.\hat{\varphi}\right|_{\left[v_{0}, u_{0}\right]}=\left.\varphi\right|_{\left[v_{0}, u_{0}\right]}$ and Lemma 4.1 we infer

$$
C_{q}(\hat{\varphi}, 0)=\delta_{q, d_{m}} \mathbb{Z} \quad \forall q \in \mathbb{N}_{0}
$$

while the coercivity of $\hat{\varphi}$ entails (cf. [18, Proposition 6.64])

$$
C_{q}(\hat{\varphi}, \infty)=\delta_{q, 0} \mathbb{Z}, \quad q \in \mathbb{N}_{0}
$$

Now, if $K(\hat{\varphi})=\left\{0, u_{0}, v_{0}, u_{1}\right\}$ then the Morse relation (2.1) written for $t=-1$ and (4.5)-(4.8) would imply

$$
(-1)^{d_{m}}+2(-1)^{0}+(-1)^{1}=(-1)^{0},
$$

which is impossible. Thus, there exists a further point $v_{1} \in K(\hat{\varphi}) \backslash\left\{0, u_{0}, v_{0}, u_{1}\right\}$. Lemma 3.1. combined with (3.9), shows that $v_{1}$ turns out to be a nodal solution of (4.1) that lies in $\left[v_{0}, u_{0}\right]$. Standard regularity arguments provide $v_{1} \in C^{1}(\bar{\Omega})$. Finally, reasoning as before one achieves $v_{1} \in \operatorname{int}_{C^{1}(\bar{\Omega})}\left(\left[v_{0}, u_{0}\right]\right)$.

An immediate application of this result produces both constant-sign and nodal solutions to the problem

$$
\begin{cases}-\Delta u=\lambda u-g(x, u) & \text { in } \Omega, \\ \frac{\partial u}{\partial n}+\beta(x) u=0 & \text { on } \partial \Omega,\end{cases}
$$

where $\lambda>0$ while $g: \Omega \times \mathbb{R} \rightarrow \mathbb{R}$ denotes a function such that $g(\cdot, 0)=0$ and $g(x, \cdot)$ belongs to $C^{1}(\mathbb{R})$ for every $x \in \Omega$, while $g(\cdot, t)$ and $g_{t}^{\prime}(\cdot, t)$ are measurable for all $t \in \mathbb{R}$. 
Theorem 4.2. Let $\lambda \in\left(\lambda_{m}, \lambda_{m+1}\right]$ for some $m \geq 2$ and let $\left(\mathrm{g}_{2}\right)$ of Theorem 3.5 be satisfied with $p=2$. If, moreover,

$\left(\mathrm{g}_{5}\right)\left|g_{t}^{\prime}(x, t)\right| \leq b_{3}\left(1+|t|^{r-2}\right)$ in $\Omega \times \mathbb{R}$, where $2 \leq r<2^{*}$,

$\left(\mathrm{g}_{6}\right) g_{t}^{\prime}(x, 0)=\lim _{t \rightarrow 0} \frac{g(x, t)}{t}=0$ uniformly with respect to $x \in \Omega$, and

$\left(\mathrm{g}_{7}\right) \int_{0}^{\xi} g(x, t) d t \geq \frac{\lambda-\lambda_{m+1}}{2} \xi^{2}$ for all $(x, \xi) \in \Omega \times \mathbb{R}$,

then the same conclusion of Theorem 4.1 holds true concerning (4.9).

The sign condition $\operatorname{tg}(x, t) \geq 0,(x, t) \in \Omega \times \mathbb{R}$, clearly forces $\left(\mathrm{g}_{8}\right)$. So, Theorem 4.2 basically extends [22, Theorem 14]. For $\beta \equiv 0$, cf. also [8, Theorem 3.7], [11, Section 4], and the references given there.

\section{Acknowledgement}

Work performed under the auspices of GNAMPA of INDAM.

\section{References}

[1] S. Aizicovici, N. S. Papageorgiou, and V. Staicu, Degree Theory for Operators of Monotone Type and Nonlinear Elliptic Equations with Inequality Constraints, Mem. Amer. Math. Soc. 196 (2008).

[2] S. Aizicovici, N. S. Papageorgiou, and V. Staicu, Existence of multiple solutions with precise sign information for superlinear Neumann problems, Ann. Mat. Pura Appl. 188 (2009), 679-710.

[3] W. Allegretto and Y. X. Huang, A Picone's identity for the p-Laplacian and applications, Nonlinear Anal. 32 (1998), 819-830.

[4] D. Averna, S. A. Marano, and D. Motreanu, Multiple solutions for a Dirichlet problem with p-Laplacian and set-valued nonlinearity, Bull. Austral. Math. Soc. 77 (2008), 285-303, and amended version.

[5] G. Barletta, P. Candito, and D. Motreanu, Constant sign and sign-changing solutions for quasilinear elliptic equations with Neumann boundary conditions, J. Convex Anal. 21 (2014), 53-66. 
[6] S. Carl and S. Heikkilä, Fixed Point Theory in Ordered Sets and Applications, Springer, New York, 2011.

[7] S. Carl, V.K. Le, and D. Motreanu, Nonsmooth Variational Problems and their Inequalities, Springer Monogr. Math., Springer, New York, 2007.

[8] G. D'Aguì, S. A. Marano, and N. S. Papageorgiou, Multiple solutions to a Neumann problem with equi-diffusive reaction term, Discrete Contin. Dyn. Syst. Ser. S 5 (2012), 765-777.

[9] D. G. de Figueiredo and J.-P. Gossez, Strict monotonicity of eigenvalues and unique continuation, Comm. Partial Differential Equations 17 (1992), 339-346.

[10] L. Gasiński and N. S. Papageorgiou, Nonlinear Analysis, Ser. Math. Anal. Appl. 9, Chapman and Hall/CRC Press, Boca Raton, 2006.

[11] L. Gasiński and N. S. Papageorgiou, Multiple solutions for a class of nonlinear Neumann eigenvalue problems, Comm. Pure Appl. Anal. 13 (2014), 1491-1512.

[12] An Lê, Eigenvalue problems for the p-Laplacian, Nonlinear Anal. 64 (2006), 10571099.

[13] S. A. Marano and N. S. Papageorgiou, Constant-sign and nodal solutions for a Neumann problem with $p$-Laplacian and equi-diffusive reaction term, Topol. Methods Nonlinear Anal. 28 (2011), 233-248.

[14] S. A. Marano and N. S. Papageorgiou, On a Neumann problem with p-Laplacian and noncoercive resonant nonlinearity, Pacific J. Math. 253 (2011), 103-123.

[15] S. A. Marano and N. S. Papageorgiou, Constant-sign and nodal solutions to a Dirichlet problem with p-Laplacian and nonlinearity depending on a parameter, Proc. Edinburgh Math. Soc. 57 (2014), 521-532.

[16] J. Mawhin and M. Willem, Critical Point Theory and Hamiltonian Systems, Appl. Math. Sci. 74, Springer-Verlag, Berlin, 1989.

[17] E. Michael, Continuous selections. I, Ann. of Math. 63 (1956), 361-382.

[18] D. Motreanu, V. V. Motreanu, and N. S. Papageorgiou, Topological and Variational Methods with Applications to Nonlinear Boundary Value Problems, Springer, New York, 2013. 
[19] D. Motreanu and M. Tanaka, Multiple existence results of solutions for quasilinear elliptic equations with a nonlinearity depending on a parameter, Ann. Mat. Pura Appl. 193 (2014), 1255-1282.

[20] D. Mugnai and N. S. Papageorgiou, Resonant nonlinear Neumann problems with indefinite weights, Ann. Sc. Norm. Sup. Pisa Cl. Sci. 11 (2014), 729-788.

[21] E. H. Papageorgiou and N. S. Papageorgiou, A multiplicity theorem for problems with the p-Laplacian, J. Funct. Anal. 244 (2007), 63-77.

[22] N. S. Papageorgiou and V. D. Radulescu, Multiple solutions with precise sign for nonlinear parametric Robin problems, J. Differential Equations 256 (2014), 24492479 .

[23] K. Perera and M. Schechter, Topics in Critical Point Theory, Cambridge Tracts in Math. 198, Cambridge Univ. Press, Cambridge, 2013.

[24] J. Su, Semilinear elliptic boundary value problems with double resonance between two consecutive eigenvalues, Nonlinear Anal. 48 (2002), 881-895.

[25] J. L. Vázquez, A strong maximum principle for some quasilinear elliptic equations, Appl. Math. Optim. 12 (1984), 191-202. 